\title{
Risk of opioid-related aberrant behaviors predicted by Opioid Risk Tool among cancer patients receiving opioid analgesics
}

\section{So Yeon Oh ( $\nabla$ manic2db@gmail.com )}

Pusan National University Yangsan Hospital https://orcid.org/0000-0002-2562-9257

\section{Kwonoh Park}

Pusan National University Yangsan Hospital

\section{Su-Jin Koh}

Ulsan University Hospital

Jung Hun Kang

Gyeongsang National University

\section{Myung Hee Chang}

National Health Insurance Service Ilsan Hospital

\section{Kyung Hee Lee}

Yeungnam University School of Medicine and College of Medicine

\section{Research Article}

Keywords: Opioid risk tool, opioid-related disorders, narcotic-related disorders, cancer pain

Posted Date: April 22nd, 2021

DOl: https://doi.org/10.21203/rs.3.rs-368890/v1

License: (1) This work is licensed under a Creative Commons Attribution 4.0 International License. Read Full License 


\section{Abstract}

Purpose: The risk of opioid-related aberrant behavior (OAB) in Korean cancer patients has not been previously evaluated. The purpose of this study was to investigate the Opioid Risk Tool (ORT) in Korean cancer patients receiving opioid treatment.

Methods: Data were obtained from a multicenter, cross-sectional, nationwide observational study regarding breakthrough cancer pain. The study was conducted in 33 South Korean institutions from March 2016 to December 2017. Patients were eligible if they had cancer-related pain within the past 7 days, which was treated with strong opioids in the previous 7 days.

Results: We analyzed ORT results of 947 patients. Only one patient in each sex $(0.2 \%)$ was classified as high risk for OAB. In patients with an ORT score of 1 or higher, the score primarily represented positive responses for personal history of depression, personal or family history of alcohol abuse, or 16-45 years age range. These patients had more severe average and worst pain scores and used rescue analgesics more frequently than patients with ORT scores of 0 . The proportion of moderate- or high-risk patients according to ORT was lower in patients receiving low doses of long-acting opioids than in those receiving high doses $(2.0 \%$ vs. $6.6 \%, p=0.030)$. Moderate or high risk was more frequent when ORT was completed in an isolated room than in an open, busy place $(2.7 \%$ vs. $0.6 \%, p=0.091)$.

Conclusion: The risk of OAB measured by ORT was very low in cancer patients receiving strong opioids for analgesia.

\section{Introduction}

Opioid analgesics are the most important drugs for controlling cancer pain. Nevertheless, their potential for dependency, misuse, and addiction has long been a major concern. Recently, the prescription of opioids has soared worldwide. Because of cultural conventions, opioid usage has been traditionally low in East Asia, but it has increased rapidly in recent years [1, 2]. In a large-scale cohort study, the proportion of opioid users in South Korea increased six to nine times from 2002 to 2015 [3]. Moreover, opioid-related chemical coping was $21 \%$ among South Korean patients receiving long-term opioid therapy for chronic non-cancer pain in a cross-sectional study [4]. Thus, concerns about opioid-related aberrant behavior $(\mathrm{OAB})$ are now greater than ever.

The rate of opioid misuse is approximately $21-29 \%$ among patients with chronic pain, according to a systematic review of studies conducted in North America and Europe [5]. To prescreen high-risk patients, many tools have been developed for predicting the risk of OAB [6]. The Opioid Risk Tool (ORT), Current Opioid Misuse Measure (COMM), and Patient Medication Questionnaire (PMQ) are common risk assessment tools [7-9]. Although no controlled study has directly compared the performance of these tools, ORT is the most widely used. In the original study describing the use of ORT, approximately $66 \%$ and $24 \%$ of patients with chronic pain were classified as having a moderate and high risk of aberrant opioid use, respectively. With respect to cancer patients, the risk of OAB varies considerably according to 
cancer type and treatment situation. Moderate to high risk of OAB predicted by ORT has been reported in approximately $15-43 \%$ of cancer patients [10-12]. In a recent study using different screening tools, $10-$ $39 \%$ of cancer patients receiving supportive care were noted to be at risk of $O A B[13,14]$.

In this paper, we describe the ORT results of a large multicenter, nationwide survey regarding breakthrough cancer pain in South Korean patients. The results of the entire study population will be published elsewhere [15]. In this study, we used ORT to evaluate the risk of OAB in patients receiving opioids to control cancer pain.

\section{Methods}

Study design

This study is a subgroup analysis of a multicenter, cross-sectional, nationwide observational study about breakthrough cancer pain. The study was conducted in 33 South Korean institutions from March 2016 to December 2017. After receiving approval from the KCSG Protocol Review Committee, this study was also approved by the Institutional Review Boards of each participating center (Pusan National University Yangsan Hospital IRB No. 04-2016-002).

\section{Patient eligibility}

Patients were eligible if they met the following criteria: 1) aged 19 years or older, 2) histologicallydiagnosed cancer, 3) current or previous anti-cancer treatment (surgery, radiation, or systemic therapy) or palliative care, 4) cancer-related pain within 7 days before the date of written informed consent, 5) use of strong opioids within 7 days before the date of written informed consent, and 6) cognitive function sufficient to read and understand the informed consent form and study questionnaires.

\section{Data acquisition}

We first collected details about cancer status and treatment history from the medical records of patients who provided written informed consent. Patients were requested to complete a questionnaire about breakthrough cancer pain, as well as the Barrier Questionnaire and ORT. If patients were unable to complete the questionnaires on their own, their caregiver was permitted to record the responses. In the absence of a caregiver, the clinical research nurse recorded the responses. The identity of the person providing assistance was recorded, as was the physical location where the questionnaires were completed.

Statistical analyses

Continuous variables are summarized as mean \pm standard deviation (SD) or median (minimum, maximum). Frequency, percentage, and cumulative percentage are presented for categorical variables. To compare two continuous variables, the two-sample t-test was used for normally distributed data, and the Mann-Whitney U test was used for non-normally distributed variables. Chi-square test or Fisher's exact 
test was used to compare categorical variables between groups. All statistical analyses were performed using IBM SPSS Statistics version 26 and Microsoft Excel 2019.

\section{Ethical statement}

The authors are accountable for all aspects of this work. All authors are ensuring that questions related to the accuracy or integrity of the work are appropriately investigated and resolved. The protocol was performed in accordance with the principles of the Declaration of Helsinki (as revised in 2013) and the Good Clinical Practice Guidelines defined by the International Conference on Harmonization. All patients provided written informed consent before enrollment.

\section{Results}

\section{Patient characteristics}

The full analysis set of this study consisted of 956 patients. The characteristics of these patients are summarized in Table 1. The majority of patients had stage IV cancer and a fair performance status, and most had received anti-cancer treatment within the previous 4 weeks. Nine patients did not complete the ORT. Therefore, we analyzed the ORT results of 947 patients.

Opioid Risk Tool results

The ORT results are presented in Table 2. In men, scores above 0 were primarily derived from positive responses for depression, personal or familial history of alcohol abuse, and age within the 16 to 45 years range. In women, the majority of scores above 0 were derived from positive responses for depression and the 16 to 45 years age range. Drug abuse and a history of preadolescent sexual abuse or psychological disease other than depression were extremely rare in both sexes. Figure 1 is a color-coded depiction of the number of positive responses for each ORT item as an easy-to-read presentation of the results. The mean total ORT score was very low in both men and women, with a median value of 0 in both sexes. The distribution of total scores for each sex is presented in Table 3. Most males were classified as low risk, and $18(3.3 \%)$ were considered moderate risk. Likewise, most females were classified as low risk, and only three $(0.7 \%)$ were classified as moderate risk. Only one subject $(0.2 \%)$ in each sex was considered high risk according to ORT. The proportion of moderate- or high-risk patients was higher in men than in women $(3.5 \%$ vs. $0.9 \%, p=0.011$ by Fisher's exact test).

Opioid Risk Tool scores and pain intensity

Because the vast majority of subjects had a 0 ORT score, we further analyzed the patients who answered 'YES' on any item of the ORT, except age ( $n=99,10.5 \%)$. These patients had a higher average pain intensity score during the past week than those with a 0 ORT score (mean \pm SD: $4.04 \pm 2.10$ vs. $3.22 \pm$ $1.94, p<0.001$ by t-test). Furthermore, patients with moderate or severe pain according to the average 1week pain intensity score were more likely to answer 'YES' to at least one ORT item than those with weak pain intensity ( $17.0 \%$ vs. $7.7 \%, p<0.001$ by chi-squared test) (Table 4$)$. Likewise, patients with an ORT 
score of 1 or more had a higher maximal pain intensity during the previous 1 week than those with a 0 ORT score (mean \pm SD: $6.66 \pm 2.53$ vs. $6.00 \pm 2.47, p=0.013$ by t-test). Patients with an ORT score of at least 1 also used short-acting opioids more frequently to control breakthrough pain, compared to patients with an ORT score of 0 ( $2.5 \pm 1.6$ times/day vs. $2.0 \pm 1.6$ times/day, $p=0.013$ by t-test). Additionally, pain interfered with the enjoyment of life in the past 24 hours more in patients with an ORT of least 1 than in those with an ORT score of 0 (Brief Pain Inventory-Short Form, Korean version, scores: $6.7 \pm 2.9$ vs. $6.1 \pm$ $2.9, p=0.037$ by t-test).

Opioid utilization and Opioid Risk Tool score

The daily dose of total long-acting opioids was converted to oral morphine equivalents (OME) for each patient. The mean and median OMEs were $93.1 \pm 246.8 \mathrm{mg}$ and $60 \mathrm{mg}(0,6300)$, respectively. The mean daily OME of patients classified as moderate or high risk according to ORT was not statistically significantly higher than that of the low risk patients (mean \pm SD: $143.1 \pm 194.6 \mathrm{mg}$ vs. $91.9 \pm 247.9 \mathrm{mg}$, $p=0.228$ by t-test). However, the proportion of patients who were classified as moderate or high risk was lower in the low-dose group than in the high-dose group ( $2.0 \%$ vs. $6.6 \%, p=0.030$ by Fisher's exact test) (Table 5).

\section{Circumstances during Opioid Risk Tool completion}

We further investigated whether the circumstances when completing ORT affected the ORT results. First, we compared the scores of self-completed ORT $(n=420)$ with the scores of ORTs completed with the assistance of a caregiver $(n=56)$ or study staff $(n=471)$. Although all high-risk patients were in the selfcompleted group, there was no statistically significant difference in ORT scores according to whether the ORT was completed alone or with assistance ( $p=0.111$, Likelihood ratio Chi-Square). Second, we tested whether the environment where patients completed the ORT correlated with ORT scores. ORT scores tended to be lower when ORT was completed in an open, busy space $(n=155)$ than when it was completed in a quiet, isolated room $(n=801)$ (moderate or high risk in open, busy location vs. quiet, isolated location: $0.6 \%$ vs. $2.7 \%, p=0.091$ by Fisher's exact test, Supplementary Table 1 ).

\section{Discussion}

In this study, we investigated the risk of OAB by using ORT to survey cancer patients who were already receiving strong opioids for pain control. To our knowledge, this is the first study to investigate ORT results in Korean patients with cancer. In this study, the risk of OAB according to ORT was very low in cancer patients receiving strong opioids for cancer-related pain. Among almost 1,000 patients, only one man and one woman were classified as high risk. This proportion $(0.2 \%)$ was lower than the percentages previously reported in the literature. Nearly $88 \%$ of males and $82 \%$ of females had ORT scores of 0 . Of those patients with an ORT score of 1 or higher, the score primarily reflected positive responses for a history of depression, alcohol abuse, and age with the 16 to 45 years range. 
In this study, we demonstrated several new findings. Patients with an ORT score of 1 or more had higher average and worst pain intensities, reported more interference with their enjoyment of life because of pain, and used more short-acting opioids for breakthrough pain, when compared to patients with an ORT score of 0 . Additionally, high-dose opioid users tended to be classified as moderate or high risk according to ORT more frequently than low-dose opioid users. We also noted that the place where ORT is completed may influence the results. Patients were more frequently classified as moderate or high risk when ORT was completed in a quiet, independent environment than when it was compared in a busy, open space. This result suggests that if researchers use ORT in a future study, it should be completed in a quiet, independent location.

Although ORT scores seemed be associated with pain intensity, it is not clear that patients with more severe pain have a higher risk of OAB. However, the likelihood that patients with a higher risk for OAB may demand more opioids cannot be ruled out. Conversely, consideration should be given to the possibility that positive response on some of the ORT items may reflect a lower pain threshold. Many studies have reported substantial genetic influences in drug addiction, reflecting the hereditability of addiction [16-18]. This genetic trait may influence both ORT scores and opioid demands. Because this study was crosssectional, we cannot confirm causality and should interpret this finding with caution.

It is worth considering why our population had an overall low risk of OAB. One possibility is that the actual risk is low in patients with cancer pain. In the past, it was generally accepted that cancer patients had an extremely low likelihood of opioid addiction or OAB [19-21]. However, more recent studies have reported that the rate of opioid addiction or aberrant behavior is increasing in this patient population. The prevalence of $O A B$ was reported as low as $7.7 \%$ and as high as $43 \%$ in these more recent studies [10-14, 22]. Thus, it is likely that the overall prevalence of $O A B$ in cancer patients is no longer low. Another possible explanation for the overall low risk observed in the current study was that patients may have wanted to be perceived as 'good' by their doctors $[23,24]$. Consequently, they may have concealed elements of their past history that they thought would have little effect, or even a negative impact, on their treatment. Although statistical significance was not achieved, no one was classified as high risk when ORT was completed with the assistance of medical staff. All high-risk patients were in the group of patients who completed ORT by themselves. A third, and most important, possibility is that ORT is not a suitable risk predicting tool in cancer patients who are already receiving opioid analgesics. ORT was originally developed to predict the probability of aberrant behaviors indicative of abuse in patients with chronic pain [7]. The original study presented no information about the primary diagnosis of the subjects. In cancer patients, the risk of OAB has been reported to vary considerably, from $10 \%$ to $40 \%$ [10-14]. In a study comparing ORT of cancer patients and heart failure patients, the proportion of moderate- to highrisk patients was higher in cancer patients (39\% versus $23 \%$ ), although the difference was not statistically significant [25]. Some studies have reported that the predictive performance of ORT is relatively poor in various populations of patients with chronic non-cancer pain. The ORT scores of chronic non-cancer pain patients using a physician-administered ORT in a tertiary care pain clinic were quite different from those reported in the original ORT study [26]. In another study of patients with chronic pain, neither patientgenerated nor physician-generated ORT was predictive of moderate-to-severe aberrant drug-related 
behavior [27]. Thus, ORT may not be an appropriate predictor for OAB, and some investigators have tried to simplify and improve the performance of this risk assessment tool [28].

This study has several limitations, First, this study was not specifically designed to evaluate OAB; instead, ORT was completed as part of a study regarding cancer pain. Thus, because the study's endpoint was not targeted for ORT, one must be careful about drawing conclusions regarding OAB in cancer patients. As discussed above, another screening tool for OAB may have performed better in this patient population, which could be explored in future studies. Second, the cancer stage was heterogenous in our study population. At least 115 patients (12\%) were at stage I to III, indicating that they may have had no residual tumor. Opioid prescribing may differ substantially between patients with and without viable tumor tissue. Third, ORT was completed under varying circumstances, according to the study timeline and study sites, and environment and location may affect ORT results. For example, ORT contains personal information, which may be embarrassing to patients. Therefore, it is probably more appropriate to complete this survey in an isolated space.

In summary, the risk of aberrant behavior measured by ORT was very low in Korean cancer patients receiving strong opioids for pain control. Patients with at least one ORT risk item (i.e., ORT score of 1 or higher) had higher average and worst pain intensities, reported more interference with enjoyment of life because of pain, and used short-acting opioids more frequently. The circumstances under which ORT was completed may have influenced the results. In future studies evaluating the risk of $O A B$, it is recommended that risk predicting tools be selected according to the subjects' characteristics.

\section{Declarations}

\section{Funding}

This study was supported by a grant from the National R\&D Program for Cancer Control, Ministry of Health and Welfare, Republic of Korea (1720150) and TEVA Korea. The research was also supported by the Korean Cancer Study Group (KCSG) and the KCSG data center.

\section{Conflicts of Interest}

All authors reported that there were no conflicts of interest related to this study.

\section{Availability of data and material}

Data and material of this study could be shared upon request by corresponding author.

\section{Code availability}

Not applicable

\section{Authors' contributions}


Conception and design: All authors; Administrative support: SK and KHL; Provision of study materials or patients: all authors; Collection and assembly of data: SYO, KP and SK; Data analysis and interpretation: SYO, KP, and JHK; Manuscript writing: SYO; Final approval of manuscript: all authors.

\section{Ethics approval}

This study was approved by the Institutional Review Boards of every participating center (Pusan National University Yangsan Hospital IRB No. 04-2016-002).

\section{Consent to participate}

All patients provided written, informed consent before enrollment. Only patients who signed consent form participated in the study.

\section{Consent for publication}

All authors agree to publish this paper on Supportive Care in Cancer.

\section{References}

1. Choi AGM, Ko JH, Kim JS, Son ES, Kwon KH. Recent Statistics and Risk Factors of Fentanyl Buccal Tablet Related Opioid Dependence Tendency at a Tertiary Hospital in Korea. 2019;36:476-85

2. Atlas on substance use (2010) resources for the prevention and treatment of substance use disorders. World Health Organization, Geneva

3. Oh TK, Jeon Y-T, Choi JW (2019) Trends in chronic opioid use and association with five-year survival in South Korea: a population-based cohort study. Br J Anaesth 123:655-663

4. Castañeda AM, Lee C-S, Kim Y-C, Lee D, Moon JY. Addressing Opioid-Related Chemical Coping in Long-Term Opioid Therapy for Chronic Noncancer Pain: A Multicenter, Observational, Cross-Sectional Study. 2018;7:354

5. Vowles KE, McEntee ML, Julnes PS, Frohe T, Ney JP, van der Goes DN (2015) Rates of opioid misuse, abuse, and addiction in chronic pain: a systematic review and data synthesis. Pain 156:569-576

6. Ducharme J, Moore S (2019) Opioid Use Disorder Assessment Tools and Drug Screening. Missouri medicine 116:318-324

7. Webster LR, Webster RM (2005) Predicting aberrant behaviors in opioid-treated patients: preliminary validation of the Opioid. Risk Tool 6:432-442

8. Butler SF, Budman SH, Fernandez KC, Houle B, Benoit C, Katz N, Jamison RN. Development and validation of the current opioid misuse measure. 2007;130:144-56

9. Adams LL, Gatchel RJ, Robinson RC, Polatin P, Gajraj N, Deschner M, Noe C. Development of a selfreport screening instrument for assessing potential opioid medication misuse in chronic pain patients. 2004;27:440-59 
10. Barclay JS, Owens JE, Blackhall LJ (2014) Screening for substance abuse risk in cancer patients using the Opioid Risk Tool and urine drug screen. Support Care Cancer 22:1883-1888

11. Garcia C, Lefkowits C, Pelkofski E, Blackhall L, Duska LR (2017) Prospective screening with the validated Opioid Risk Tool demonstrates gynecologic oncology patients are at low risk for opioid misuse. Gynecol Oncol 147:456-459

12. Ma JD, Horton JM, Hwang M, Atayee RS, Roeland EJ (2014) A Single-Center, Retrospective Analysis Evaluating the Utilization of the Opioid Risk Tool in Opioid-Treated Cancer Patients. Journal of Pain Palliative Care Pharmacotherapy 28:4-9

13. Yennurajalingam S, Edwards T, Arthur JA, Lu Z, Najera J, Nguyen K, Manju J, Kuriakose L, Wu J, Liu D, Williams JL, Reddy SK, Bruera E (2018) Predicting the risk for aberrant opioid use behavior in patients receiving outpatient supportive care consultation at a comprehensive cancer center. Cancer 124:3942-3949

14. Yasin JT, Leader AE, Petok A, Garber G, Stephens B, Worster B (2019) Validity of the screener and opioid assessment for patients with pain-revised (SOAPP-R) in patients with cancer. J Opioid Manag 15:272-274

15. Koh SLK, Oh S, Kang J, Koo D, Yun S, Chang M, Park J, Lee M, Choi Y, Chang J. Abstracts for MASCC/ISOO Annual Meeting 2019. In: Supportive Care in Cancer; 2019 2019/06/01; San Francisco, CA; p. 1-302

16. Kendler KS, Jacobson KC, Prescott CA, Neale MC. Specificity of Genetic and Environmental Risk Factors for Use and Abuse/Dependence of Cannabis, Cocaine, Hallucinogens, Sedatives, Stimulants, and Opiates in Male Twins. 2003;160:687-95

17. Wang SC, Chen YC, Lee CH, Cheng CM (2019) Opioid Addiction, Genetic Susceptibility, and Medical Treatments: A Review. Int J Mol Sci 20:4294

18. Tsuang MT, Lyons MJ, Meyer JM, Doyle T, Eisen SA, Goldberg J, True W, Lin N, Toomey R, Eaves L. Co-occurrence of abuse of different drugs in men: the role of drug-specific and shared vulnerabilities. 1998;55:967-72

19. Evans PJ. Narcotic addiction in patients with chronic pain. 1981;36:597-602

20. Schug SA, Zech D, Grond S, Jung H, Meuser T, Stobbe B. A long-term survey of morphine in cancer pain patients. 1992;7:259-66

21. Højsted J, Sjøgren P (2007) Addiction to opioids in chronic pain patients: A literature review. Eur J Pain 11:490-518

22. Passik SD, Kirsh KL, McDonald MV, Ahn S, Russak SM, Martin L, Rosenfeld B, Breitbart WS, Portenoy RK. A pilot survey of aberrant drug-taking attitudes and behaviors in samples of cancer and AIDS patients. 2000;19:274-86

23. Jadad AR, Rizo CA, Enkin MW (2003) I am a good patient, believe it or not. BMJ 326:1293-1295

24. Sointu E (2017) 'Good' patient/'bad' patient: clinical learning and the entrenching of inequality. Sociol Health IIIn 39:63-77 
25. Freeman G, Barclay J (2017) Comparison of the Risk for Substance Abuse in Heart Failure and Cancer Patient Populations Using the Opioid Risk Tool and Urine Drug Screen (SA508D). J Pain Symptom Manage 53:397-398

26. Lakha SF, Louffat AF, Nicholson K, Deshpande A, Mailis-Gagnon A (2014) Characteristics of Chronic Noncancer Pain Patients Assessed with the Opioid Risk Tool in a Canadian Tertiary Care Pain Clinic. Pain Med 15:1743-1749

27. Witkin LR, Diskina D, Fernandes S, Farrar JT, Ashburn MA (2013) Usefulness of the opioid risk tool to predict aberrant drug-related behavior in patients receiving opioids for the treatment of chronic pain. J Opioid Manag 9:177-187

28. Cheatle MD, Compton PA, Dhingra L, Wasser TE, O'Brien CP (2019) Development of the Revised Opioid Risk Tool to Predict Opioid Use Disorder in Patients with Chronic Nonmalignant Pain. J Pain 20:842-851

\section{Tables}

Table 1. Patient characteristics 


\begin{tabular}{|c|c|}
\hline Characteristic & Value $(\mathrm{N}=956)$ \\
\hline Age (years) & $63(27,91)$ \\
\hline \multicolumn{2}{|l|}{ Sex } \\
\hline Male & $542(56.7)$ \\
\hline Female & $414(43.3)$ \\
\hline Time from initial diagnosis (months) & $15(0,384)$ \\
\hline \multicolumn{2}{|l|}{ Relapse } \\
\hline No & $632(66.1)$ \\
\hline Yes, relapsed & $315(32.9)$ \\
\hline Unknown & $9(0.94)$ \\
\hline Time to relapse (months, $\mathrm{n}=315$ ) & $12(0,164)$ \\
\hline \multicolumn{2}{|l|}{ Primary cancer diagnosis } \\
\hline Colorectal & $129(13.5)$ \\
\hline Lung & $111(11.6)$ \\
\hline Breast & $107(11.2)$ \\
\hline Stomach & $100(10.5)$ \\
\hline Pancreas & $93(9.7)$ \\
\hline Other & $416(43.5)$ \\
\hline \multicolumn{2}{|l|}{ Stage } \\
\hline 1 & $10(1.0)$ \\
\hline 2 & $31(3.2)$ \\
\hline 3 & $74(7.7)$ \\
\hline 4 & $808(84.5)$ \\
\hline Unknown & $33(3.5)$ \\
\hline \multicolumn{2}{|l|}{ ECOG PS } \\
\hline 0 & $60(6.3)$ \\
\hline 1 & $605(63.3)$ \\
\hline 2 & $190(19.9)$ \\
\hline 3 & $59(6.2)$ \\
\hline 4 & $18(1.9)$ \\
\hline
\end{tabular}

Page $11 / 18$ 


\begin{tabular}{|ll|}
\hline $\begin{array}{l}\text { Unknown } \\
\text { Recent anti-cancer treatment (past 4 weeks) }\end{array}$ & $24(2.5)$ \\
Yes & $666(69.7)$ \\
No & $290(30.3)$ \\
Type of recent anti-cancer treatment $(\mathrm{n}=666)^{\mathrm{a}}{ }^{\mathrm{a}}$ & \\
Chemotherapy & $638(66.7)$ \\
Adjuvant & $33(5.2)$ \\
Neoadjuvant & $9(1.4)$ \\
Palliative & $592(92.8)$ \\
Unknown & $4(0.6)$ \\
Radiotherapy & $79(8.3)$ \\
Surgery & $6(0.6)$ \\
\hline
\end{tabular}

Values are number (percentage) or median (minimum, maximum).

a Overlap was permitted.

ECOG Eastern Cooperative Oncology Group

Table 2. Opioid Risk Tool results according to sex 


\begin{tabular}{|c|c|c|}
\hline ORT items and total scores & $\begin{array}{l}\text { Male } \\
(n=538)\end{array}$ & $\begin{array}{l}\text { Female } \\
(n=409)\end{array}$ \\
\hline \multicolumn{3}{|c|}{ Family history of substance abuse } \\
\hline \multicolumn{3}{|l|}{ Alcohol } \\
\hline No & $521(96.13)$ & $401(96.9)$ \\
\hline Yes & $16(3.0)$ & $8(1.9)$ \\
\hline NA & $5(0.9)$ & $5(1.2)$ \\
\hline \multicolumn{3}{|l|}{ Illegal drugs } \\
\hline No & 537 (99.1) & 409 (98.8) \\
\hline NA & $5(0.9)$ & $5(1.2)$ \\
\hline \multicolumn{3}{|l|}{ Prescription drugs } \\
\hline No & $531(98.0)$ & $408(98.6)$ \\
\hline Yes & $6(1.1)$ & $2(0.5)$ \\
\hline NA & $5(0.9)$ & $4(1.0)$ \\
\hline \multicolumn{3}{|c|}{ Personal history of substance abuse } \\
\hline \multicolumn{3}{|l|}{ Alcohol } \\
\hline No & $518(95.6)$ & $406(98.1)$ \\
\hline Yes & $19(3.5)$ & $3(0.7)$ \\
\hline NA & $5(0.9)$ & $5(1.2)$ \\
\hline \multicolumn{3}{|l|}{ Illegal drugs } \\
\hline Yes & $536(98.9)$ & $409(98.8)$ \\
\hline No & $1(0.2)$ & $0(0)$ \\
\hline NA & $5(0.9)$ & $5(1.2)$ \\
\hline \multicolumn{3}{|l|}{ Prescription drugs } \\
\hline No & $535(98.7)$ & $408(98.6)$ \\
\hline Yes & $2(0.4)$ & $2(0.5)$ \\
\hline NA & $5(0.9)$ & $4(1.0)$ \\
\hline \multicolumn{3}{|l|}{ Age (16-45 years) } \\
\hline Not 16-45 & $522(96.3)$ & $385(93.00)$ \\
\hline
\end{tabular}




\begin{tabular}{|llc|} 
N6-45 & $15(2.8)$ & $24(5.8)$ \\
\hline History of preadolescent sexual abuse & $5(0.9)$ & $5(1.2)$ \\
No & $537(99.1)$ & $408(98.6)$ \\
Yes & $0(0)$ & $1(0.2)$ \\
\hline NA & $5(0.9)$ & $5(1.2)$ \\
\hline Psychological disease & & \\
Attention deficit, obsessive-compulsive disorder, bipolar, schizophrenia \\
No & $533(98.3)$ & $408(98.6)$ \\
Yes & $4(0.7)$ & $1(0.2)$ \\
NA & $5(0.9)$ & $5(1.2)$ \\
\hline Depression & & \\
\hline No & $519(95.8)$ & $371(89.6)$ \\
\hline Yes & $19(3.5)$ & $38(9.2)$ \\
\hline NA & $4(0.7)$ & $5(1.2)$ \\
\hline Total score & & $0.2 \pm 0.7$ \\
Mean \pm SD & $0.3 \pm 1.2$ & \\
\hline Median (minimum, maximum) & $0(0,12)$ & \\
\hline
\end{tabular}

Data for ORT items are numbers (percentage).

$N A$ not available, $O R T$ Opioid Risk Tool, $S D$ standard deviation

Table 3. Distribution of Opioid Risk Tool total scores according to sex 


\begin{tabular}{|lll|}
\hline Total ORT score & \multicolumn{2}{l|}{ Number (\%) } \\
\cline { 2 - 3 } & Male & Female \\
\hline $\mathbf{0}$ & $475(87.6)$ & $339(81.9)$ \\
\hline $\mathbf{1}$ & $23(4.2)$ & $61(14.7)$ \\
\hline $\mathbf{2}$ & $1(0.2)$ & $3(0.7)$ \\
\hline $\mathbf{3}$ & $20(3.7)$ & $2(0.5)$ \\
\hline $\mathbf{4}$ & $9(1.7)$ & $3(0.7)$ \\
\hline $\mathbf{5}$ & $3(0.6)$ & 0 \\
\hline $\mathbf{6}$ & $5(0.9)$ & 0 \\
\hline $\mathbf{7}$ & $1(0.2)$ & 0 \\
\hline $\mathbf{8}$ & 0 & $1(0.2)$ \\
\hline $\mathbf{1 2}$ & $1(0.2)$ & 0 \\
\hline NA & $4(0.7)$ & $5(1.2)$ \\
\hline Sum & $542(100)$ & $414(100)$ \\
\hline
\end{tabular}

NA not available, ORT opioid risk tool

Table 4. Association between average pain intensity in the previous week and Opioid Risk Tool scores

\begin{tabular}{|c|c|c|c|c|c|}
\hline \multirow{3}{*}{$\begin{array}{l}\text { ORT score } \\
0\end{array}$} & \multicolumn{3}{|c|}{ Average pain intensity } & \multirow{2}{*}{\multicolumn{2}{|c|}{$P$ value }} \\
\hline & \multirow{2}{*}{$\begin{array}{l}\text { Weak } \\
627\end{array}$} & \multicolumn{2}{|c|}{ Moderate or severe } & & \\
\hline & & 230 & 857 & & .001 \\
\hline$\geq 1$ & 52 & 47 & 99 & & \\
\hline Sum & 679 & 277 & 956 & & \\
\hline
\end{tabular}

ORT opioid risk tool

a Based on the number of ORT items with a 'YES' answer, excluding the age range item.

${ }^{b}$ Chi-squared test

Table 5. Association between Opioid Risk Tool risk classification and dose of long-acting opioids for background pain 


\begin{tabular}{|llllll|}
\hline \multirow{2}{*}{ Dose of long-acting opioids $^{\mathrm{a}}$} & \multicolumn{2}{l}{ ORT risk } & Sum & P value $^{\mathbf{b}}$ \\
\cline { 2 - 5 } & Low & Moderate or high & & \\
\hline Low & 862 & 18 & 880 & 0.030 \\
\hline High & 71 & 5 & 76 & \\
\hline Sum & 933 & 23 & 956 & \\
\hline
\end{tabular}

ORT, Opioid Risk Tool.

a Total daily dose of long-acting opioids (i.e., extended-release, controlled-release, and slow-release forms) for background pain converted to oral morphine equivalents (OME). High dose was defined as daily OME of $200 \mathrm{mg}$ or higher.

${ }^{b}$ Fisher's exact test

Figures 


\begin{tabular}{|c|c|c|}
\hline No. of 'YES' & Male & Female \\
\hline Age (16-45 years) & 15 & 24 \\
\hline F.Hx: alcohol & 16 & 6 \\
\hline F.Hx: illegal drugs & 0 & 0 \\
\hline F.Hx: prescription drugs & 6 & 2 \\
\hline Hx: alcohol & 19 & 3 \\
\hline Hx: illegal drugs & 1 & 0 \\
\hline Hx: prescription drugs & 2 & 2 \\
\hline Hx: sexual abuse & 0 & 1 \\
\hline Psychological disorders & 4 & 1 \\
\hline Depression & 19 & 38 \\
\hline
\end{tabular}

\section{Figure 1}

The number of patients who answered 'YES' to each Opioid Risk Tool item are displayed in a different color according to the size of number. The higher the number, the darker the color. F.Hx family history, $\mathrm{Hx}$ personal history.

\section{Supplementary Files}


This is a list of supplementary files associated with this preprint. Click to download.

- Supplementarydatav1.0.docx 